\title{
BMJ Open Evaluating the effectiveness of care integration strategies in different healthcare systems in Latin America: the EQUITY-LA II quasi-experimental study protocol
}

\author{
María-Luisa Vázquez, ${ }^{1}$ Ingrid Vargas, ${ }^{1}$ Jean-Pierre Unger, ${ }^{2}$ Pierre De Paepe, ${ }^{2}$ \\ Amparo Susana Mogollón-Pérez, ${ }^{3}$ Isabella Samico, ${ }^{4}$ Paulette Albuquerque, ${ }^{5}$ \\ Pamela Eguiguren, ${ }^{6}$ Angelica Ivonne Cisneros, ${ }^{7}$ Mario Rovere, ${ }^{8}$ \\ Fernando Bertolotto ${ }^{9}$
}

To cite: Vázquez M-L, Vargas I, Unger J-P, et al. Evaluating the effectiveness of care integration strategies in different healthcare systems in Latin America: the EQUITY-LA II quasiexperimental study protocol. BMJ Open 2015;5:e007037. doi:10.1136/bmjopen-2014007037

- Prepublication history for this paper is available online. To view these files please visit the journal online (http://dx.doi.org/10.1136/ bmjopen-2014-007037).

Received 26 February 2015 Revised 28 April 2015 Accepted 29 April 2015

CrossMark

For numbered affiliations see end of article.

Correspondence to Dr María-Luisa Vázquez; mlvazquez@consorci.org

\section{ABSTRACT}

Introduction: Although fragmentation in the provision of healthcare is considered an important obstacle to effective care, there is scant evidence on best practices in care coordination in Latin America. The aim is to evaluate the effectiveness of a participatory shared care strategy in improving coordination across care levels and related care quality, in health services networks in six different healthcare systems of Latin America.

Methods and analysis: A controlled before and after quasi-experimental study taking a participatory action research approach. In each country, two comparable healthcare networks were selected-intervention and control. The study contains four phases: (1) A baseline study to establish network performance in care coordination and continuity across care levels, using (A) qualitative methods: semi-structured interviews and focus groups with a criterion sample of health managers, professionals and users; and (B) quantitative methods: two questionnaire surveys with samples of 174 primary and secondary care physicians and 392 users with chronic conditions per network. Sample size was calculated to detect a proportion difference of $15 \%$ and $10 \%$, before and after intervention ( $\alpha=0.05 ; \beta=0.2$ in a two-sided test); (2) a bottom-up participatory design and implementation of shared care strategies involving micro-level care coordination interventions to improve the adequacy of patient referral and information transfer. Strategies are selected through a participatory process by the local steering committee (local policymakers, health care network professionals, managers, users and researchers), supported by appropriate training; (3) Evaluation of the effectiveness of interventions by measuring changes in levels of care coordination and continuity 18 months after implementation, applying the same design as in the baseline study; (4) Crosscountry comparative analysis.

Ethics and dissemination: This study complies with international and national legal stipulations on ethics. Conditions of the study procedure were approved by each

\section{Strengths and limitations of this study}

- The results of this research will support national decision-makers by providing the first available evidence on best practices in health care integration in Latin America.

- The participation of a wide range of Latin American countries with different health systems will provide a better understanding of the influence of contextual factors on health care coordination.

- This research will empower decision-makers to better translate knowledge into policies and clinical practices by involving key stakeholders in a participatory process of action research from the beginning of the project.

- One limitation might be that the implementation of interventions depends on the willingness of subjects and selected health services networks to participate and to lead the process. Fluid communication with them will be encouraged to overcome this.

country's ethical committee. A variety of dissemination activities are implemented addressing the main stakeholders. Registration No.257 Clinical Research Register of the Santa Fe Health Department, Argentina.

\section{INTRODUCTION}

Health services fragmentation is considered to be one of the main obstacles to attaining effective healthcare outcomes in many Latin American health systems. It produces difficulties in access to care, poor technical quality, discontinuity of care and inefficiency. ${ }^{1}$ These weaknesses are most evident in the care of patients with chronic conditions that require 
the coordination of multiple health professionals and care settings. ${ }^{2}{ }^{3}$ Owing to the demographic and epidemiological changes seen in Latin America in recent years, ${ }^{3}$ the care of patients with chronic conditions is becoming an increasingly relevant challenge to address.

In this context of care fragmentation, the policy challenge is to improve care integration. ${ }^{124}$ Many countries have promoted the development of integrated healthcare networks (IHN), defined as a network of organisations that provides or arranges to provide a coordinated continuum of services to a defined population and is willing to be held clinically and fiscally accountable for the health status and outcomes of this population. ${ }^{15}$ These countries include, among others, Colombia, ${ }^{6}$ Brazil,${ }^{7}$ Chile, ${ }^{8}$ Mexico, ${ }^{9}$ Uruguay $^{10}$ and Argentina. ${ }^{11}$

IHN initiatives have received strong support from international organisations, ${ }^{4}{ }^{12-14}$ despite the scarce evidence of their impact. Results from a previous study on IHNs in Colombia and Brazil (Equity-LA) reveal poor perceived coordination across care levels, limited implementation of care coordination mechanisms and significant hindrances to care coordination, related both to the health system model and the organisation of the healthcare network (I Vargas, AS Mogollón-Pérez, P De Paepe, et al. Barriers to care coordination in market-based and decentralized public health systems: a qualitative study in healthcare networks of Colombia and Brazil. Health Policy Plan 2014; [submitted]). However, similar evidence from other Latin American health systems is scarce,${ }^{15-17}$ and questions concerning best practices in care integration for the Latin American context, and the structural and organisational reforms required to improve IHN performance, remain unexplored. ${ }^{1}$

The expected results of this research projectEquity-LA II, which builds on those of Equity-LA, will support national and international decision-makers by providing evidence of best practices in care integration in different health systems in Latin America, with particular reference to two chronic diseases (diabetes and chronic obstructive pulmonary disease (COPD). It will also contribute to advancing the state of the art by generating new knowledge on the impact of IHN reforms on coordination across care levels and, ultimately, quality of care. The project will achieve this by expanding the scope of the research in Colombia and Brazil and incorporating other Latin American (LA) countries -Chile, Mexico, Argentina and Uruguay-thus representing a large array of health systems and IHNs.

\section{Care coordination and quality as outcomes of IHN performance}

In theory, the integration of healthcare delivery should contribute to more efficient, more equitable and higher quality health services ${ }^{18}$ through improvements in care coordination, continuity and access. ${ }^{19}$ Consequently, IHN performance analysis should take into account both final outcomes (efficiency, equity and quality of care) and intermediate outcomes (care coordination, continuity of care and access to healthcare), as is proposed in the Equity-LA conceptual framework. ${ }^{19}$ This aims to analyse IHN performance taking the following factors into account: the internal processes developed by IHNs to achieve their objectives; the macro-level and micro-level contexts in which IHNs perform (including the type of health system and its objectives regarding equity of access, efficiency and quality of care, and care coordination), and the social actors involved in the care coordination process, that is, healthcare professionals, managers and users (figure 1).

Care coordination is defined here as the harmonious connection of the different services needed throughout the care continuum to provide care for a patient, in order to achieve a common objective without conflicts. ${ }^{20}$ Three types can be distinguished: informational coordination, or the transfer and use of the patient clinical information needed to coordinate activities between providers; clinical management coordination, or the provision of care in a sequential and complementary way; ${ }^{21}$ and administrative coordination, or the coordination of patient access through the continuum of services according to their needs. ${ }^{22}$ While clinical care integration is considered the highest degree of coordination, the term continuity of care refers to how individual patients experience the coordination of services, and it is defined as the degree to which patients experience care over time as coherent and linked. ${ }^{21}$ Finally, following the IOM definition, ${ }^{23}$ quality of care is defined as the degree to which health services for individuals and populations increase the likelihood of desired health outcomes and are consistent with current professional knowledge. ${ }^{21} \mathrm{~A}$ review of the literature shows that there is no consensus on how best to approach the analysis of coordination and continuity across care levels and its relationship to quality of care. However, the empirical evidence indicates that a lack of coordination across care levels is one of the most frequent causes of poor quality-duplication of diagnostic testing, perilous polypharmacy, inappropriate referrals, and confusion about conflicting care plans, ${ }^{24}{ }^{25}$ suggesting that an appropriate and necessary way to address quality improvement is to advance in care coordination. To contribute to progress beyond the state of the art, Equity-LA II will develop and apply research tools, building on and further developing the Equity-LA conceptual framework.

\section{Interventions to improve the performance of IHNs}

The evidence is conclusive on the organisational elements that are critical for IHN performance. ${ }^{5}{ }^{19}$ These elements, which are included in the Equity-LA conceptual framework (figure 1), are as follows: (A) a shared vision of the system's goals and strategies across the network; (B) methods for resource allocation that align health services incentives with the global objectives of the network; (C) an organic structure with mechanisms that enhance communication between health professionals involved in the care process; (D) a common culture and leadership with values oriented at teamwork, 


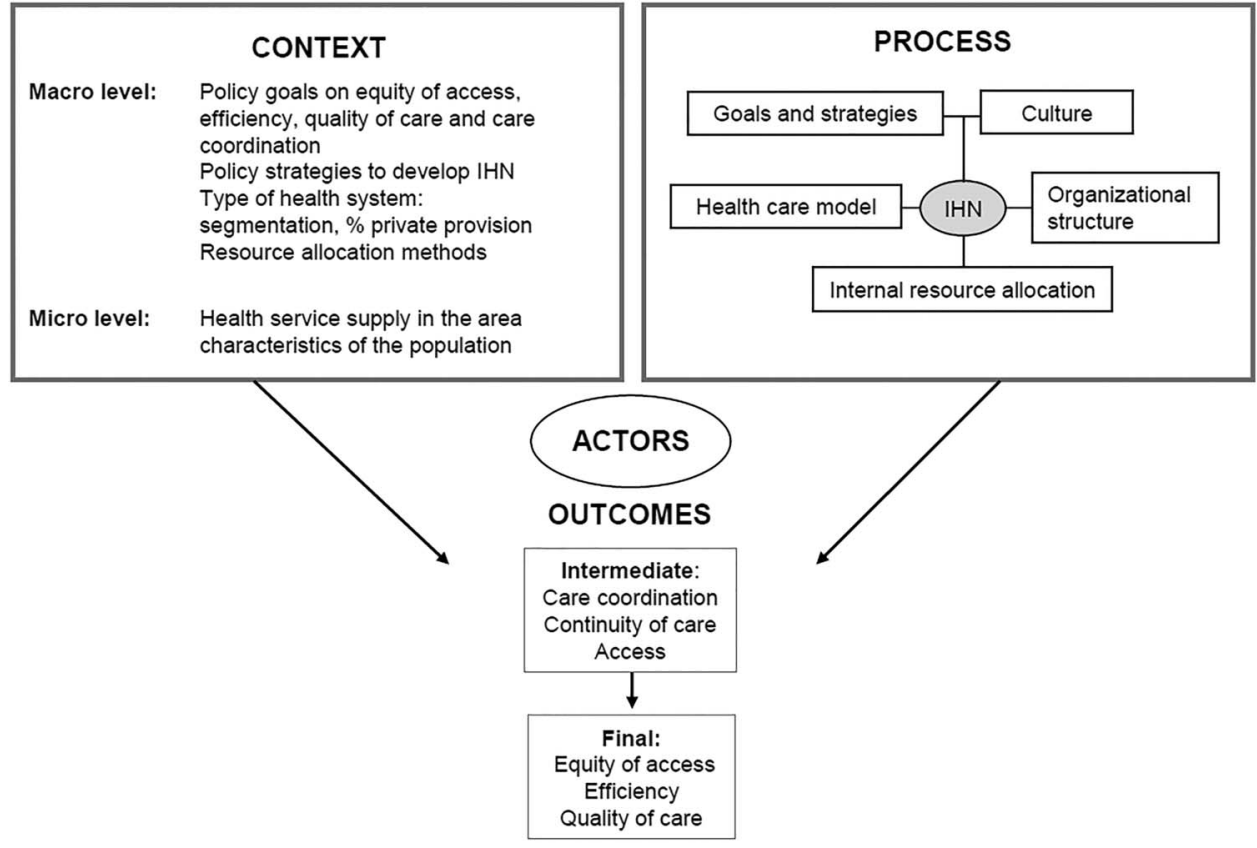

Figure 1 Framework for the analysis of Integrated Health Care Networks (IHN) and its impact. Source: Modified from Vázquez et al. ${ }^{19}$

collaboration and best performance; and (E) a healthcare model based on primary healthcare.

To address these critical elements, an array of interventions at different levels can be considered ${ }^{26}:$ (1) macrolevel: policies and regulatory mechanisms to develop IHN; (2) meso-level: IHNs' strategic plans and coordination mechanisms for managerial functions (eg, integrated management strategies, shared management committee); (3) micro-level: the introduction of a single care coordination mechanism or a combination of mechanisms in a comprehensive programme (eg, shared care strategy, disease and case management programmes).

The literature recommends the implementation of integration strategies at the micro-level led by healthcare professionals from the beginning of the IHN configuration process ${ }^{26-29}$ This argument is based on one of the main weaknesses detected in the development of such initiatives: the tendency of IHN managers to prioritise strategic discourses and organisational changes and to ignore actions aimed at improving clinical management. ${ }^{30}$ Although macro-level and meso-level strategies facilitate clinical integration, ${ }^{5}$ mechanisms for care coordination seem to be more effective in enhancing collaboration between professionals of different care levels by creating spaces for communication and mutual understanding, such as multidisciplinary healthcare teams across levels or shared clinical sessions. ${ }^{31}$ Micro-level coordination strategies will be the focus of Equity-LA II.

Evidence on effective interventions to improve integration of care in Latin America and other contexts

Evaluations of interventions to improve coordination across care levels have mainly been carried out in the
USA and Europe. These show, first, that interventions involving multiple strategies are more successful than those which employ a single strategy ${ }^{29} 32$ and, second, that despite the methodological weaknesses associated with the studies conducted, there is a certain amount of evidence to suggest a connection between specific strategies and improved care outcomes, appropriate health services utilisation and technical efficiency. ${ }^{29} \quad 33 \quad 34$ Shared care strategies that encompass multidisciplinary groups for care coordination, disease management programmes and case management ${ }^{34}$ have been shown to improve care outcomes in specific population groups, such as patients with psychiatric illnesses, stroke victims and patients with diabetes, with improvements being noted in mortality rates and hospital readmissions, among other outcomes. ${ }^{34}$ However, the effects of these interventions on other groups of patients are unknown, as are the effects of other interventions proposed for improving care coordination. It also remains to be seen whether the results obtained for evaluations in the North American and European contexts are applicable to other contexts such as Latin America.

There have been few evaluations to date of best practices in care integration in the Latin American context. Existing studies have so far been conducted at the national level and have focused on specific populations - mainly mother and child health. ${ }^{35}{ }^{36}$ Moreover, most studies have concentrated on the evaluation of a single mechanism, addressing only one dimension of care coordination, such as the implementation of information technology in healthcare settings ${ }^{37} 38$ or the expert system (case reviews, clinical sessions, etc) in mental healthcare. ${ }^{39}{ }^{40}$ Evidence is also scarce with regard to 
the factors and actors that influence the effectiveness of the introduction of these micro-level interventions in different health systems and settings in middle-income countries.

\section{Need for adequate research design and approaches in testing care integration interventions}

The fact that the existing evidence is scarce on the effectiveness of organisations' strategies for improving coordination, and even scarcer with regard to improvements in the final outcomes of efficient use of resources and quality of care, is mainly due to underdeveloped theories and research designs, over-reliance on experimental designs and the lack of independent evaluations. ${ }^{33}$ To address this gap, the need to develop more quasi-experimental research has been pointed out. $^{33} 34{ }^{41}$ With the type of design, observed differences in performance are assumed to be due to the intervention, since controlled before and after studies protect against secular trends and sudden changes. ${ }^{42} 43$ Furthermore, quasi-experimental studies allow us to consider participants' preferences, which may be important in influencing their motivation and attitudes. ${ }^{42}$ These aspects may be enhanced by adopting a participatory action research (PAR) approach ${ }^{44}$ that focuses on learning, success and action, as this is a collaborative approach that builds on the strengths, values and contributions of all the actors concerned. ${ }^{45}{ }^{46}$ Thus, its strength lies in generating solutions to practical problems-in this research, strategies to address problems of care coordination across care levels-as well as in implementing those solutions. Furthermore, by systematically monitoring and reflecting on the process and outcomes of change ${ }^{47}$ it helps to bridge the gap between theory and practice in both directions: knowledge for practice and practice for knowledge. ${ }^{48}$

\section{Aims of the equity-LA II project}

The ultimate goal is to improve the effectiveness, efficiency and equity of healthcare systems, particularly in Latin America, by providing evidence on best practices in care integration, which will be translated into effective policies for varying social, political and economic contexts. The general objective is to evaluate the effectiveness of a participatory shared care strategy in improving coordination across levels of care and related quality of care in health services networks in different Latin American healthcare systems. This paper describes the study protocol and discusses the potential contributions of the project to existing knowledge.

\section{METHODS AND ANALYSIS}

Study design

The study adopts a quasi-experimental design (a controlled before and after design) for evaluating the effectiveness of a shared care strategy, with a multidisciplinary PAR approach. PAR entails the documentation of problems, achievements and the state of the art, the formulation of an analysis and action model including the design, implementation and evaluation of interventions, ${ }^{47} 49$ and the stakeholders' active participation throughout all research phases. ${ }^{50}$ To achieve the active participation of the stakeholders throughout the research process, a local steering committee was set up at the beginning of the project in each study country, representing all the stakeholders involved in the health services network: healthcare professionals, managers, users, local policymakers and researchers. The local steering committee participates in all the project phases and is in charge of the selection, design and implementation of interventions. To fulfil this key role effectively, the committees are given appropriate training in accordance with the needs identified at the beginning of the project. This combination of approaches aims to avoid a fragmented or imbalanced account of problems and solutions and ensure an equal relationship between researchers and other participants.

The study evaluates integration interventions for improving coordination across levels of care and related quality of care, both in general terms and in specific reference to two chronic conditions: type 2 diabetes and COPD. These chronic conditions have been chosen due to their increasing relevance in epidemiological terms ${ }^{51}$ and to the need of patients in both cases for lifelong and continuous medical care across care levels. ${ }^{52} 53$

\section{Study area}

Research is being carried out in the six participating Latin American middle-income countries, representing different types of healthcare systems. In each country, two comparable health services networks-one intervention and one control network-have been selected according to the inclusion criteria: (A) provision of a continuum of services including at least primary and secondary care; (B) provision of services to a defined population; (C) provision of care mainly to urban slums; (D) willingness to participate and implement designed interventions; and (E) leadership with the authority to implement designed strategies. The selected networks are: Colombia: South-Western and Southern health services networks of the District Health Department of Bogotá; Brazil: two micro-regions (3.2 and 3.3) of District III in Recife and the urban area of Caruarú in Pernambuco state; Chile: health services networks of three districts in the Southern and Northern area of Santiago; Mexico: the health services networks of Xalapa and Veracruz; Argentina: Southern and North-Western districts of Rosario; Uruguay: the Durazno/Florida and Soriano/ Río Negro districts of the Eastern region.

\section{Research phases and methods}

The project is structured in four sequential research phases which will take place over 60 months, using Campbell et al $\mathrm{s}^{54}$ model for the evaluation of complex intervention and taking the PAR approach: (1) a baseline 
Figure 2 Research phases and methods.

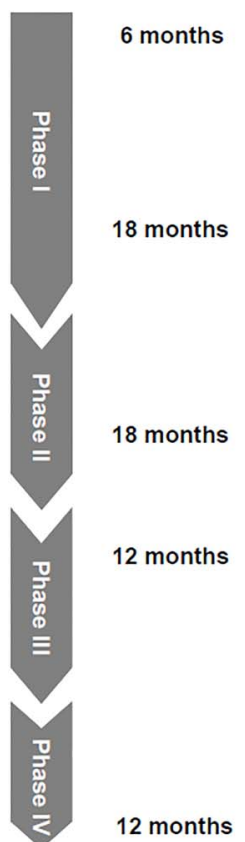

Research framework and research plan

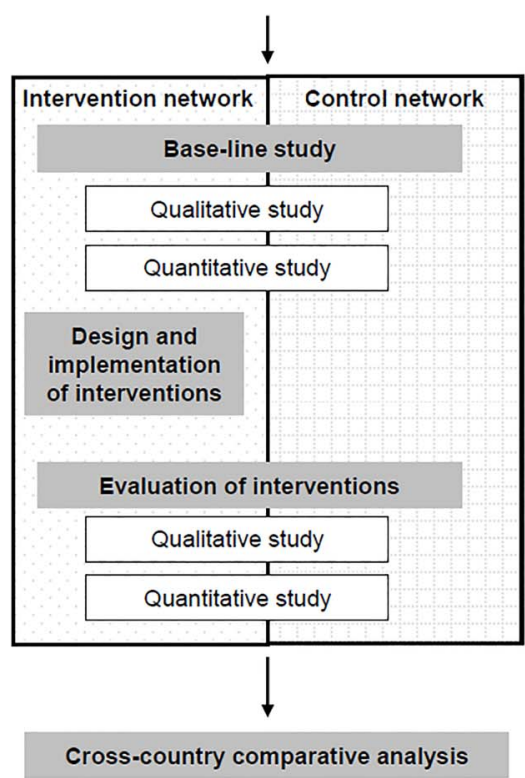

study, using both qualitative and quantitative methods, which is currently underway; (2) participatory selection, design and implementation of interventions; (3) evaluation of results; and a (4) cross-country comparative analysis (figure 2).

\section{Phase 1: A baseline study}

During the first 6 months of this phase, the research framework and research plan were finalised. The next step is to evaluate the performance of the intervention and control health services networks with respect to coordination across levels of care and continuity of care, and related quality of care, a process which is currently underway. The evaluation employs a combination of qualitative and quantitative methods in order to obtain baseline information on the provision of care to the general population and patients with chronic conditions (including diabetes and COPD):

A. Qualitative study. Individual interviews and focus groups have been carried out in order to investigate coordination across levels of care and related quality of care, and to explore the experiences across the care continuum of patients with chronic conditions, including type 2 diabetes and COPD. Individual interviews provide an insight into the perspectives of healthcare workers, users and managers and administrative personnel and focus groups are used to explore certain important issues emerging from the individual interviews. Criterion sampling ${ }^{55}$ was used to select informants, applying the following inclusion criteria: (1) healthcare professionals of different care levels (primary, secondary/tertiary care) providing care for patients from the study areas, with at least 6 months' experience. To provide a variation in discourse, the following criteria were taken into account: age, sex, care level, type of work contract and specialty; (2) users (and their caregivers) with chronic conditions (including diabetes and COPD) who had used the first care level and used or tried to use the secondary care level in the past 6 months. The following maximum variation criteria were applied: age (over 18) and severity of illness. The final sample size was reached by saturation of information. Topic guides were developed with one common and one specific section for each informant group. Participants were identified through healthcare provider records. Approval was sought for their participation in focus groups and individual interviews both by telephone and home visits. All focus groups and individual interviews were recorded and transcribed. The qualitative data analysis is still underway. Transcriptions are being analysed using text-coding software. A thematic content analysis ${ }^{56}$ is being conducted. Data have been segmented by network, informant group and themes. The categories used have been generated from a mix of the topic guide and those emerging from the data. The analysis takes the gender perspective into account. Themes are being identified, coded, recoded and classified, identifying common patterns by looking at regularities, and convergences and divergences in the data, through a process of constant comparison, going back and forth between the data. To ensure data quality, results are being triangulated using different research methods and relying on different informant groups and multiple analysts with different backgrounds and an in-depth knowledge of qualitative methods, the research topic and its context. ${ }^{57}$ The members of the local steering committee are also participating in the data analysis. In the different 
analytical stages, preliminary results are presented to the members of the steering committee during meetings conducted for this purpose and their feedback is then taken into account for further analysis. They are involved as part of the participatory process, so that they are given the chance to not only familiarise themselves with the data but also to influence the analytical process. Moreover, the results of the baseline study should serve to guide the selection of the most appropriate intervention in each context.

B. Quantitative studies. Two questionnaire surveys are being conducted: (1) primary and secondary/tertiary doctors will be surveyed to determine their perceptions of the degree of coordination across levels of care, as well as their knowledge and use of existing care coordination mechanisms and influencing factors; and (2) users of 18 years of age or over with chronic conditions (including type 2 diabetes and COPD), who were attended to in primary care and secondary care for the same condition in the 3 months prior to the survey, will be surveyed to determine their perceptions and experiences of continuity of care and factors that influence on them. A sample of doctors and users will be selected in both networks under investigation in the baseline and evaluation phases. A list of doctors will be drawn up based on the information provided by the centres. Since the number of doctors in most networks is relatively small, all primary and secondary care physicians are being invited and encouraged by the research team to participate in the survey. Patients fulfilling the criteria are being selected in the waiting room of the primary care health facilities. Since the flow of patients in primary care centres is relatively low, all the users in the waiting room of each primary care centre in the network will be approached. The surveys have started between May or June, depending on the country, and are expected to past from 6 weeks to 2 months each, depending on the response of doctors and the flow of patients. The sample size has been calculated taking into account the controlled before and after design of the study. With respect to health professionals, a sample size of 174 for each network (and phase) was estimated in order to ensure the detection of a $15 \%$ variation in professionals' perception of coordination of care, both between phases and between networks. For the users' survey, a sample size of 388 patients per network (and phase) was estimated in order to ensure the detection of a $10 \%$ variation in patients' perception of continuity of care, both between phases and between networks. In both cases, sample size is calculated on the basis of $80 \%$ power and a confidence level of $95 \%$. Descriptive analyses will be carried out, and $\mathrm{t}$ tests and $\chi^{2}$ tests will be used to compare differences in outcomes between intervention and control networks. In addition, a multivariable analysis will be conducted using statistical software in order to explore the effects of potential explanatory variables on the outcome. The analysis of the baseline study on network performance with respect to coordination across levels of care and continuity of care, and related quality of care will be completed by combining the preliminary results of the qualitative study with those of the quantitative study.

\section{Phase 2: Participatory design and implementation of interventions}

In all the study countries, the preliminary results of the qualitative baseline study reveal limited coordination between the primary and secondary care levels. This is reflected in reports of poor clinical information transfer, inappropriate referrals of patients to secondary care and the follow-up of patients in secondary care rather than in primary care when appropriate. The local steering committee of each country will be in charge of selecting, designing and implementing the interventions aimed at addressing the problems identified in the baseline study. They will also be responsible for recruiting and training participants in their countries throughout the process. The members will undergo a process of learning by doing and will be coached by the researchers in putting these activities into practice in their network. Additional data to those obtained in the baseline study may be collected (through interviews with key informants, review of records, etc) in order to hone the design of the interventions and monitor the process of implementation.

\section{Design of interventions}

A shared care strategy will be developed in each country through a participatory process led by the local steering committee. The strategy will be designed to address the main difficulties of the referral system, especially to improve the adequacy of the referral and counterreferral of patients and the transfer of clinical information between primary and secondary care services. The shared care strategy involves the joint participation of primary and secondary care physicians and will combine a series of care coordination mechanisms. The selected strategies will be conceived to motivate health professionals to participate on a voluntary basis. Any activity taken on should be incorporated into their regular duties, so the proposed interventions should be affordable for local authorities. The following list of potential mechanisms is not prescriptive since the final selection of interventions will depend on the results of the baseline study and the decision made by each local steering committee.

A. Programming strategies: the development of an expert system (continuous medical training, alternatives to traditional consultations such as case reviews, shared clinical sessions, and other training mechanisms such as hospital rotation for primary care doctors), rationalisation tools for clinical decisionmaking in handling COPD and diabetes (clinical guidelines, design of care maps or clinical pathways). 
B. Feedback strategies: placement of care managers and liaison professionals, the implementation of in-service supervision and individual coaching, the development of multidisciplinary working groups to enhance communication between health professionals, the fostering of informal channels of communication, and an adequate use of clinical information systems.

Since one of the factors that influence the use of care coordination mechanisms by professionals is their degree of involvement in the selection, design, implementation and evaluation processes, the interventions will: (A) be bottom up, meaning that health professionals in the network will play a central role in introducing the changes ${ }^{58}{ }^{59}$; (B) be based on staff training through techniques such as in-service demonstrations, case reviews and audit-oriented observations ${ }^{59}$; and (C) ensure a balance between the rationalisation of clinical decision-making and the individual health professional's therapeutic autonomy. ${ }^{59}$

\section{Implementation of interventions}

The shared care strategy will be implemented in the intervention network in each country and, if proven effective, introduced in the control network (comparative arm) at the end of the country study, in order to prevent changes occurring too early and thereby hindering the detection of differences. The previously trained local steering committee will be in charge of monitoring the intervention process in each country. On introducing the selected intervention, particular attention will be paid to its rate of uptake and stability. Their constant monitoring will facilitate the adaptation of the intervention to achieve optimal effectiveness if, for example, the proposed intensity or duration of the intervention is found to be unacceptable to participants. This cyclical process represents the classical spiral of PAR. ${ }^{54}$ The process will be based on learning by doing, which will provide sustainability after the end of the project.

\section{Phase 3: evaluation of interventions}

In this phase, the effectiveness of the shared care strategy implemented will be evaluated, recognising its limitations and identifying factors that determine its applicability in different contexts. As well as measuring the impact of the interventions, factors that may hinder or enable their implementation will be identified for each setting. This evaluation, which also combines qualitative and quantitative methods, will follow the same design as the one used in the baseline study. The main analysis dimensions of coordination, continuity and related quality of care are those used in the baseline study.

Two intracountry comparisons will be made: (1) before and after in each health services network, to compare performance in both the intervention and control networks before and after the intervention period; (2) intervention-control comparison, to measure differences in performance between the intervention and control networks. Evaluations will analyse improvements in coordination across levels of care and related quality of care, as well as contextual factors influencing the strategies and their results. To compare differences in outcomes (the levels of coordination and continuity of care) between the baseline and the evaluation phases, and within and between networks, the following analyses of survey data will be performed: (A) a bivariate analysis using the $\chi^{2}$ test and (B) multivariate analyses, adjusted by explanatory variables using logistic or linear regression models, according to the dependent variable analysed.

The analysis of network performance with respect to coordination across levels of care and continuity of care will be completed by combining the preliminary results of the qualitative study with those of the quantitative study. It will also include feedback for professionals in order to improve their clinical practice skills and use of care coordination mechanisms.

\section{Phase 4: Cross-country comparative analysis and} generation of recommendations and tools for translating research into policy

Comparisons will be made at two levels: (1) with the other Latin American countries analysed; and (2) with international studies in which networks have been evaluated, such as those from Catalonia (Spain). ${ }^{26}$ The comparative analysis will result in a better understanding of the link between different interventions and their outcomes, and the associated factors in each health system and healthcare setting. Results will be used to identify and document best practices in care integration, and to establish the best tools and guidelines for translating evidence on best practice into policies.

\section{Capacity building}

During the four phases, capacity building will be carried out in three main areas: (1) capacity building of policymakers for the planning, management and organisation of health systems by involving key stakeholders from the beginning of the project and generating evidence-based tools for the development of policy and research dissemination; (2) strengthening of research capacity of all involved institutions in health system research through the specific training of junior researchers and the exchange of knowledge and experiences among senior researchers; and (3) improvement of health professionals' knowledge and skills in coordination across care levels and quality of care through in-service training programmes.

\section{ETHICS AND DISSEMINATION \\ Ethical issues}

The development and execution of the project fully complies with all current international conventions and declarations, ${ }^{60}$ EU legislation, ${ }^{61}{ }^{62}$ national legislation, ${ }^{63-80}$ ethical regulations, data protection laws and 
the professional code of conduct of all the countries involved. Conditions of study procedure, risk and benefit evaluation, confidence and privacy and informed consent were approved by the ethical committees in the participating countries. In addition, confidentiality agreements were signed with all participating institutions. Free and informed consent will be obtained from every interviewee, after being informed that participation is voluntary and that they are free to refuse to participate without any negative consequence. Data will be coded and processed in such a way that the individual origin cannot be identified, and appropriately stored. The project and the data processing will comply with the European Union Data Protection Legislation and national legislation.

In accordance with the current legislation and regulations in each country, the project has been submitted and approved by the corresponding ethical committees in the eight countries involved: Clinical Research Ethics Committee, CEIC-Parc de Salut Mar, Spain (Comité Ético de Investigación Clínica, CEIC-Parc de Salut Mar), approved on 13 March 2013; Institutional Research Board, Institute of Tropical Medicine, Belgium, approved on 8 August 2013; Research Ethics Committee, School of Medicine and Health Sciences, University of El Rosario, Colombia (Comité de Ética en Investigación, Escuela de Medicina y Ciencias de la Salud, Universidad del Rosario), approved on 18 July 2013; Ethics Committee for Research on Humans, Institute of Integrative Medicine Prof. Fernando Figueira, Brazil (Comitê de Ética em Pesquisas em Seres Humanos, Instituto de Medicina Integral Professor Fernando Figueira), approved on 23 January 2014; Bioethics Committee and Southern Metropolitan Health Service Ethics Committee, Chile (Comité de Bioética and Comité Ético del Servicio de Salud Metropolitano Sur), approved on 11 December 2013; Health Services Research Ethics Committee of Veracruz State, Mexico (Comité de Ética en Investigación de los Servicios de Salud del Estado de Veracruz), approved on 6 November 2013; Research Ethics Committee, Public Health Department of Rosario Municipality, Argentina (Comité de Ética en Investigación, Secretaría de Salud Pública de la Municipalidad de Rosario), approved on 29 May 2013; Registration No. 257 in the Clinical Research Register of the Research Ethics Committee of the Santa Fe Province Health Department, Argentina (Registro de Investigaciones Clínicas del Comité Provincial de Bioética); Ethics Committee, School of Nursing, University of the Republic of Uruguay (Comité de Ética de la Facultad de Enfermería de la Universidad de la República), approved on 12 February 2014.

\section{Dissemination}

The dissemination of research results is also a transversal component of the project to ensure that the findings are used to inform policy and practice and disseminated to the greater public, the following mechanisms for the management of newly generated knowledge will be considered: (1) for dissemination among the academic communities, short reports and research papers in free access peer-reviewed national and international journals and other relevant publications, as well as participation in national and international conferences; (2) to ensure that results inform policymaking: (A) a best practices report on care integration in the region and policy guidelines; (B) round tables, meetings and workshops to present key findings and policy recommendations to local and national interest groups in the six Latin American countries; (C) building networks of key contacts (academic, governmental, non-governmental, civil society, including users' organisations, etc) in the participating countries and other countries in Latin America and elsewhere, and with international agencies, such as the PAHO, World Bank, WHO, EC, etc; and (D) coordinating with pre-established networks.

\section{Author affiliations}

${ }^{1}$ Health Policy and Health Services Research Group, Health Policy Research Unit, Consortium for Health Care and Social Services of Catalonia, Barcelona, Spain

${ }^{2}$ Public Sector Care Unit, Department of Public Health, Prince Leopold Institute of Tropical Medicine, Antwerpen, Belgium

${ }^{3}$ Escuela de Medicina y Ciencias de la Salud, Universidad del Rosario, Bogotá, Colombia

${ }^{4}$ Grupo de Estudos de Gestão e Avaliação em Saúde, Instituto de Medicina Integral Prof. Fernando Figueira, Recife, Brasil

${ }^{5}$ Universidade de Pernambuco, Recife, Brasil

${ }^{6}$ Escuela de Salud Pública Salvador Allende Gossens, Universidad de Chile, Santiago de Chile, Chile

${ }^{7}$ Instituto de Salud Pública, Universidad Veracruzana, Xalapa, México

${ }^{8}$ Maestría en Salud Pública, Centro de Estudios Interdisciplinarios, Instituto de la Salud Juan Lazarte, Universidad Nacional de Rosario, Rosario, Argentina

${ }^{9}$ Facultad de Enfermería, Universidad de la República, Montevideo, Uruguay

Acknowledgements The authors are most grateful to Marta Beatriz Aller, Irene Garcia-Subirats, Sina Waibel, Hernán Vargas and Lluis Camprubí for their contributions to the study protocol; and to the European Commission Seventh Framework Programme (FP7/2007-2013) which is funding this grant agreement (no. 305197) and the previous project Equity-LA (no. 223123).

Contributors M-LV, IV, J-PU, PDP and ASM-P conceived the concept, objectives and the study design; M-LV and IV were responsible for the development of the study protocol, which received the contributions of the other authors. IV and M-LV wrote the first draft of the paper. All authors reviewed the draft versions, made contributions and approved the final version of the article. The authors alone are responsible for the content of this paper.

Funding This research project is funded by the European Commission Seventh Framework Programme (FP7/2007-2013) under grant agreement number 305197.

Competing interests None declared.

Patient consent Obtained.

Ethics approval Comité Ético de Investigación Clínica Parc de Salut Mar, Barcelona, Spain. Institute of Tropical Medicine Institutional Review Board, Antwerp, Belgium. Comité de Ética en Investigación, Escuela de Medicina y Ciencias de la Salud, Universidad del Rosario, Bogota, Colombia. Comitê de Êtica em Pesquisa em Seres Humanos do Instituto de Medicina Integral Prof. Fernando Figueira, Recife, Brazil. Comité Ètico Científico del Servicio Metropolitano Sur, Santiago de Chile, Chile. Comité de Ética en Investigación de los Servicios de Salud del Estado de Veracruz, Veracruz, Mexico. Comité de Ética en Investigación, Secretaría de Salud Pública Municipalidad de Rosario, Rosario, Argentina. Comité de Ética de la Facultad de Enfermería de la Universidad de la República, Montenvideo, Uruguay. 
Provenance and peer review Not commissioned; peer reviewed for ethical and funding approval prior to submission.

Open Access This is an Open Access article distributed in accordance with the Creative Commons Attribution Non Commercial (CC BY-NC 4.0) license, which permits others to distribute, remix, adapt, build upon this work noncommercially, and license their derivative works on different terms, provided the original work is properly cited and the use is non-commercial. See: http:// creativecommons.org/licenses/by-nc/4.0/

\section{REFERENCES}

1. Pan American Health Organization. Renewing primary health care in the Americas. Concepts, policy options and a road map for implementation in the Americas. Washington DC: OPS, 2010.

2. McAdam M. Framework of integrated care for the elderly. Ontario: Canadian Policy Research Network, 2008.

3. World Bank. Population aging: is Latin America ready? Washington DC: World Bank, 2011.

4. World Health Organization. Integration of health care delivery. Geneva: WHO 1996; Report No.: 861. Series: WHO Technical Report Series.

5. Shortell SM, Gillies RR, Anderson DA, et al. Remaking health care in America. The evolution of organized delivery systems. San Francisco: The Jossey-Bass Health Care Series, 2000.

6. Congreso de la República de Colombia. [Law 1438 of 2011, which included the reform of the General System of Social Security in Health and made other provisions]. 2011.

7. Republica Federativa do Brasil. [Decree 7508 of June 28, 2011 , which regulates Law 8080 of September 19, 1990, to provide for the organization of the Universal Health System (SUS), health planning, health care, the structure among the federation and other measures] 2011.

8. Ministerio de Salud, República de Chile. [Mission of the subsecretary of care networks]. http://web.minsal.cl/ (accessed 25 Aug 2014).

9. Secretaría de Salud, Estados Unidos Mexicanos. [Integrative model for health care (MIDAS)]. http://wwwdgpladessaludgobmx/ descargas/biblio/MIDASpdf (accessed 15 Oct 2014).

10. República Oriental del Uruguay. [Law no. 18335 of August 15, 2008, which regulates the rights and obligations of patients and users of health services]. (accessed 26 Aug 2008).

11. Ministerio de Salud, Presidencia de la Nación. [Remediar+Redes. Strengthening of health networks]. http://www.remediar.gov.ar/index. php?option=com content\&view=article\&id=16\&ltemid=20 (accessed 15 Oct 2014).

12. World Health Organization. Resolution WHA62.12 primary health care, including health system strengthening. Geneva: WHO, 2009

13. United Nations. The millennium development goals: a latin American and Caribbean perspective. Santiago de Chile: United Nations, 2005.

14. World Bank. Investing in health. World development report 1993. Oxford: Oxford University Press, 1993.

15. Herrera Vázquez MM, Rodríguez AN, Nebot AC, et al. [A network to promote health systems based on primary health care in the Region of the Americas]. Rev Panam Salud Publica 2007;21:261-73.

16. Vilaça Mendes E. [Health Care Networks]. Brasilia DF: Organização Pan-Americana de Saúde (OPAS), Conselho Nacional de Secretarios de Saúde. 2011

17. Montenegro $\mathrm{H}$, Holder R, Ramagem $\mathrm{C}$, et al. Combating health care fragmentation through integrated health service delivery networks in the Americas: lessons learned. Int J Integr Care 2011;19:5-16.

18. Banks P. Policy framework for integrated care for older people. London: King's Fund, 2004.

19. Vázquez ML, Vargas I, Unger JP, et al. Integrated health care networks in Latin America: toward a conceptual framework for analysis. Rev Panam Salud Publica 2009;26:360-7.

20. Terraza-Núñez R, Vargas I, Vázquez ML. [Coordination among healthcare levels: systematization of tools and measures]. Gac Sanit 2006;20:485-95.

21. Reid R, Haggerty J, McKendry R. Defusing the confusion: concepts and measures of continuity of healthcare. Ottawa: Canadian Health Services Research Foundation, 2002.

22. Vargas I, Mogollón-Pérez AS, Unger JP, et al. Regional-based Integrated Healthcare Network policy in Brazil: from formulation to practice. Health Policy Plan 2015;30:705-17.

23. Legido-Quigley $\mathrm{H}$, McKee $\mathrm{M}$, Nolte $\mathrm{E}$, et al. Assuring the quality of health care in the European Union. A case for action. Copenhagen: European Observatory on Health System and policies (WHO), 2008.
24. Ovretveit J. Does improving quality save money? London: Health Foundation, 2009.

25. Bodenheimer T. Coordinating care-a perilous journey through the health care system. N Engl J Med 2008;358:1064-71.

26. Vázquez ML, Vargas I. [Integrated healthcare organizations. A case study]. Barcelona: Consorci Hospitalari de Catalunya, 2009.

27. Gardner K, Yen L, Banfield M, et al. From coordinated care trials to medicare locals: what difference does changing the policy driver from efficiency to quality make for coordinating care? Int J Qual Health Care 2013;25:50-7.

28. Mooney $\mathrm{H}$. Integration of care should be at heart of NHS reform, says King's Fund. BMJ 2010;341:c6687.

29. Curry N, Ham C. Clinical and service integration: the route to improved outcomes. London: The King's Fund, 2010.

30. Vázquez ML, Vargas I, Nuño R, et al. [Integrated delivery systems and other examples of collaboration among providers. SESPAS report, 2012]. Gac Sanit 2012;26:94-101.

31. Henao D, Vázquez M, Vargas I. [Factors influencing coordination among healthcare levels according to the opinion of healthcare managers and health professionals]. Gac Sanit 2009;23:280-6.

32. Powel Davies $\mathrm{G}$, Willians AM, Larsen $\mathrm{K}$, et al. Coordinating primary health care: an analysis of the outcomes of a systematic review. Med J Aust 2008;188:S65-8.

33. Ovretveit J. Does clinical coordination improve quality and save money? London: Health Foundation, 2011.

34. McDonald KM, Sundaram V, Bravata DM, et al. Closing the quality gap: a critical analysis of quality improvement strategies. Volume 7 Care coordination. Rockville, MD: Agency for Healthcare Research and Quality, 2007.

35. Carvalho DS, Novaes HM. [Evaluation of the prenatal care program in Curitiba, Parana, Brazil: a cohort study of primigravidae]. Cad Saude Publica 2004;20:S220-30.

36. World Health Organization. Multi-country evaluation. Integrated Management of Childhood IIIness (IMCl). http://www.who.int/ imci-mce/ (accessed 15 Oct 2014).

37. Garcia PJ, Vargas JH, Caballero NP, et al. An e-health driven laboratory information system to support HIV treatment in Peru: E-quity for laboratory personnel, health providers and people living with HIV. BMC Med Inform Decis Mak 2009;9:50.

38. Insua JT. Integration of information technology (IT) for chronic diseases management in Latin America: 5 years experience, Hospital Universitario Austral (HUA), Argentina. AMIA Annu Symp Proc 2006;2006:963.

39. Figueiredo EN, Vianna LA, Peixe MB, et al. The challenge of the reference and counter-reference system in the prenatal assistance to pregnant women with infectious diseases. An Acad Bras Cienc 2009;81:551-8.

40. Pinto AG, Jorge MS, Vasconcelos MG, et al. [Matrixing support as an instrument of primary healthcare in mental health: multiple views and devices for resolution]. Cienc Saude Colet 2012;17:653-60.

41. Solberg LL. Care coordination: what is it, what are its effects and can be sustained? Family Pract 2011;28:469-70.

42. Eccles M, Grimshaw J, Campbell M, et al. Research designs for studies evaluating the effectiveness of change and improvement strategies. Qual Saf Health Care 2003;12:47-52.

43. Ukoumunne OC, Gulliford MC, Chinn S, et al. Methods for evaluating area-wide and organisation-based interventions in health and health care: a systematic review. Health Technol Assess 1999;3:iii-92.

44. Khanlou N, Peter E. Participatory action research: considerations for ethical review. Soc Sci Med 2005;60:2333-40.

45. The Public Health Agency of Canada. Guide to project evaluation: a participatory approach. Ottawa: Public Health Agency of Canada, 2001.

46. Whyte WF. Participatory action research. Thousand Oaks, CA: SAGE Publications, Inc., 1991

47. Meyer J. Qualitative research in health care. Using qualitative methods in health related action research. BMJ 2000;320:178-81.

48. Tanna NK. Action research: a valuable research technique for service delivery development. Pharm World Sci 2005;27:4-6.

49. Cornwall A, Jewkes R. What is participatory research? Soc Sci Med 1995;41:1667-76.

50. Hall JE. Professionalizing action research—a meaningful strategy for modernizing services? J Nurs Manag 2006;14:195-200.

51. Menezes AM, Pérez-Padilla R, Jardim JR, et al. Chronic obstructive pulmonary disease in five Latin American cities (the PLATINO study): a prevalence study. Lancet 2005;366:1875-81.

52. Barcelo A, Aedo C, Rajpathak S, et al. The cost of diabetes in Latin America and the Caribbean. Bull World Health Organ 2003;81:19-27.

53. ZuWallack R, Nici L. Integrated care of the COPD patient: a pulmonary rehabilitation perspective. Breath 2010;6:313-19. 
54. Campbell M, Fitzpatrick R, Haines A, et al. Framework for design and evaluation of complex interventions to improve health. BMJ 2000;321:694-6.

55. Fernández de Sanmamed MJ. [Study design and sample design in qualitative research]. In: Vázquez ML, da Silva MRF, Mogollón AS, et al. eds. [Introduction to qualitiative methods of research applied to health]. Barcelona: Universitat Autònoma de Barcelona, 2006:31-51.

56. Miles MB, Huberman AM. Qualitative data analysis: an expanded sourcebook. Thousand Oaks, California: SAGE Publications, Inc., 1994

57. Patton Q. Qualitative evaluation and research methods. London: SAGE Publications Inc., 1990.

58. Ham C, Imison C, Goodwin N, et al. Where next for NHS reforms? The case for integrated care. London: The King's Fund, 2011.

59. Unger JP, De Paepe P, Sen K, et al. International health and aid policies; the need for alternatives. Cambridge: Cambridge University Press, 2010.

60. World Medical Association. World Medical Association Declaration of Helsinki: ethical principles for medical research involving human subjects JAMA 2013;310:2191-4.

61. European Parliament and of the Council. Directive 95/46/EC of 24 October 1995, on the protection of individuals with regard to the processing of personal data and on the free movement of such data. 23 November 1995

62. European Parliament and of the Council. Directive 2001/20/EC of 4 April 2001 on the approximation of the laws, regulations and administrative provisions of the Member States relating to the implementation of good clinical practice in the conduct of clinical trials on medicinal products for human use. 1 May 2001.

63. Estados Unidos Mexicanos. Freedom of Information Act (FOIA) of June 11, 2002. 11 June 2002.

64. Cámara de Diputados del H.Congreso de la Unión, Estados Unidos Mexicanos. [General Health Law of June 6, 2014]. 2014.

65. Ministerio de Salud, República de Chile. [Technical rule no. 57 of June 4, 2001. Regulation on conducting clinical studies that use pharmaceutical products on human subjects]. 4 June 2001

66. Ministerio Secretaría General de la Presidencia, República de Chile. [Law 19628 of August 28, 1999 on protection of private life]. 28 August 1999.

67. Ministerio de Salud, República de Chile. [Law 20120 of September 22,2006 on scientific study of human subjects, the genome and prohibition of human cloning]. 22 September 2006.
68. Ministerio de Salud, República Argentina. [Resolution 008430 of October 4, 1993, which establishes scientific, technical and administrative rules for health research]. 4 October 1993.

69. Ministerio de Salud, República de Colombia. [Resolution 13437 of 1991, which provides for the constitution of Hospital Ethics Committees and adopts the Decalogue of Patients' Rights] 1991.

70. Ministerio de Salud, República Argentina. [Resolution 1480/11-MS of September 13, 2001, which approves the guide for research with human subjects]. 13 September 2011.

71. Presidencia de la Nación Argentina. [Decree 995/2000 of October 30, 2000. Law of Habeas Data]. 2 November 2000.

72. Presidencia de la Nación Argentina. [Law 17622/68 of January 25 1968, which creates the National Statistics and Census Institute]. 25 January 1968.

73. Belgian Government. Law 7th May 2004, concerning experiments on the Human Beings. 18 May 2004.

74. Ministério da Saúde, República Federativa do Brasil. [Resolution 196/96 of October 10, 1996, which approves the directives and regulatory norms for research with human subjects]. 10 October 1996.

75. Ministerio de Salud Pública, República Oriental del Uruguay. [Decree 379/008 of August 4, 2008, which regulates research with human subjects]. 14 August 2008.

76. República Oriental del Uruguay. [Law 18331 of August 18, 2008 , which regulates protection of personal information and that action of "Habeas Data"]. 18 August 2008

77. Congreso de la República de Colombia. [Statutory Law 1266 of December 31, 2008, which dictates the general provisions of Habeas Data and regulates the management of information in personal data bases, especially financial, credit-related, commercial, services and that which pertains to third countries, among other provisions]. 31 December 2008.

78. Congreso de la República de Colombia. [Law 23 of February 18 1981 , which constitutes the essential element for the development of standards regarding medical ethics]. 27 February 1981.

79. Ministerio de Justicia, España. [Royal Decree 994/1999 of June 11, which approves the regulation of security measures for automated files containing data of a personal nature]. 25 June 1999.

80. Jefatura del Estado, España. [Organic Law 15/1999 of December 13 , on the protection of personal data]. $14 \mathrm{Dec} .1999$ 